\title{
Machine Learning and Deep Learning Approaches to Analyze and Detect COVID-19: A Review
}

\author{
T. Aishwarya ${ }^{1}\left[\right.$ ] V. Ravi Kumar ${ }^{1}[0$
}

Received: 11 December 2020 / Accepted: 20 March 2021 / Published online: 20 April 2021

(c) The Author(s), under exclusive licence to Springer Nature Singapore Pte Ltd 2021

\begin{abstract}
COVID-19 also referred to as Corona Virus disease is a communicable disease that is caused by a coronavirus. Significant number of people who are tainted with this infection will have to brave and encounter moderate to severe respiratory sickness. Aged persons, sick, convalescing people and all those having underlying health complications like diabetes, chronic breathing diseases and cardiovascular diseases are bound to contract this sickness if not taken proper care of. At the current scenario, there are neither definite treatments nor inoculations against COVID-19. Nevertheless, there are numerous continuing clinical trials assessing the impending treatments and vaccines. Sensing the threatening impacts of Covid-19, researchers of computer science have started using various techniques and approaches of Machine Learning and Deep Learning to detect the presence of the disease using X-rays and CT images. The biggest stumbling block here is that there are only a few datasets available. There is also less number of experts for marking the information explicit to this new strain of infection in people. Artificial Intelligence centred tools can be designed and developed quickly for adapting the existing AI models and for leveraging the ability to modify and associating them with the preliminary clinical understanding to address the new group of COVID-19 and the novel challenges associated with it. In this paper, we look into a few techniques of Machine Learning and Deep Learning that have been employed to analyse Corona Virus Data.
\end{abstract}

Keywords Corona virus · Covid-19 · Machine learning · Deep learning · CT images · Convolutional neural network · Covid-net $\cdot$ IRCNN $\cdot$ CheXNet

\section{Introduction}

COVID-19 originated from China with the initial cases being reported in the Hubei Province. It has been frightening to the wellbeing of the public, the economy of the world as well as the stocks and trades. This respiratory disease referred to as Coronavirus, originating from SARS-CoV-2 virus has made way to a pandemic globally. This virus mimics behaviour as that of viral pneumonia and also has an

This article is part of the topical collection "Data Science and Communication" guest edited by Kamesh Namudri, Naveen Chilamkurti, Sushma S J and S. Padmashree.

T. Aishwarya

aishwarya.t@vvce.ac.in

V. Ravi Kumar

ravikumarv@vvce.ac.in

1 Vidyavardhaka College of Engineering, Mysuru, Karnataka, India increasing death rate if not treated immediately. The globally spreading coronavirus infection has become an undermining hazard for worldwide general wellbeing. This contamination, depending upon the severity of the infection causes infection of air sacs in both or one of the lung and loads it up with liquid or discharge, thus making it difficult for the patient to breathe. An analytic and diagnostic instrument used for pneumonia detection is the Chest CT. It is discovered to be exceptionally helpful in distinguishing ordinary radiographic highlights of COVID-19, particularly with dainty slices. CT images and X-rays depict some situations such as swelling of lesion symptoms, bronchiectasis which depicts the abnormal widening of the bronchi. Dissimilar blurriness in the CT images of patients offers to analyse COVID-19 conveniently [7]. It is now vital to quickly and precisely screen patients having community-acquired pneumonia (CAP) from patients having COVID-19.

As indicated by an ongoing report, its deadly rate comes to $2 \%$, second just $10 \%$ to SARS, also referred to as Severe Acute Respiratory Syndrome and 36\% of the MERS, called 
as Middle East Respiratory Syndrome [3]. It can be transmitted from individual to individual with a moderately high essential proliferation number (R0) of 2.2, and has no proficient medicines and control methodologies till now [1]. Presently, a person demonstrating respiratory defects such as fever, pneumonia, cough or dyspnea will be alluded to the final investigation trial of the test called Real Time Polymerase Chain Reaction (RT-PCR) [2]. Nonetheless, ongoing investigations show that RT-PCR has only $30 \%$ to $60 \%$ of moderately low discovery rate and repetitive tests are commonly required. In this manner, negative reports of RT-PCR tests will not preclude the chance of Covid-19 infection.

As indicated by the course of corona disease development and explosion of cases, the patients could be separated into various stages of sickness like mild, moderate, extreme and critically ill [3]. If there is any improvement in the symptom of patients, one can observe a gradual resolution of consolidation in images of computed tomography. Considering various stages of the illness, an increase of crazy-paving pattern and consolidation can be seen called the ground-glass opacity (GGO). Nevertheless, despite the advancements, it still stays as a pronounced challenge in Covid-19 screening and identification in CT images from actual cases of pneumonia. It is also difficult to differentiate them from each other because of the same radiographic features present in both of them. Images of CT for thin slice acquisition will encompass a number of slices to be checked manually. It may produce incorrect negative results specifically during the mild stage of the disease. Therefore, a computerized investigation and analysis means or methods for differentiating COVID-19 from Commonly Acquired Pneumonia, CAP, is very much desirable.

\section{ML and DL Techniques Against COVID-19}

We survey and put out various techniques of Machine Learning and Deep Learning that have been developed by various authors.

\section{D \& 3D CNN Based CT and X-ray Image Analysis for Detection of Covid-19}

The aim of the research work carried out in [4] was to design and develop an AI-based programmed Computed tomography image analysis tool. In this regard, numerous datasets were encompassed by the researchers and experiments were done to assess the development of this illness in each and every patient as time passes. This was done using a 3D volume evaluation, thereby generating the score called "Corona Score". A total set of 157 global patients CTs were considered as the testing set in this study. Subsystem A proposed typically comprises a 3-Dimensional analysis for nodules or slices of focal opacities and case volumes by means of previously existing algorithms. Subsystem B is a recently evolved $2 \mathrm{D}$ examination of each and every slice to distinguish and confine infiltrates of ground glass and bigger measured diffuse opacities as well. Also to address the supplementary disease-driven opacities, a solution was proposed on the basis of per-slice of cases. The current Lung pathology detection solutions can't be depended upon for recognizing progressively diffusing GG opacities since they are built with a focus particularly on tasks to identify the nodules.

In their second proposal of the solution, referred to as solution 'B', using a lung segmentation module, they extracted ROI, the region of interest from the lungs. The architecture called the U-Net is used for segmentation of images and training was done using $6150 \mathrm{CT}$ slices of cases having irregularities in the lungs. They have also used ResNet-50, a 50 layer deep Convolutional Neural Network architecture and the entire network has a depth of 50 layers in total. This network was pre-trained with the help of more than a million images that are obtained in the ImageNet database. The block diagram for the system used is presented in Fig. 1.

Employing the Deep-Learning image analysis system that they created, they attained outcomes of classification for Non-Coronavirus cases versus coronavirus cases. This was done for every thoracic CT studies having AUC at about 0.996 on Chinese controlled datasets and patients who were infected. The probable working points are sensitivity of $98.2 \%$, specificity of $92.2 \%$ for high sensitivity point and 98\% for specificity for high specificity point. The "Corona score" that they suggest processes the advancement of disease by the passing of time.

Various other researchers have also contributed to the designing of deep learning models to detect the presence of Covid-19 disease. In [15], the authors have proposed a hybrid 2D/3D CNN-centered approach for Covid-19 screening referred to as Hybrid-Covid. They have used X-rays of the chest as they are proven to detect respiratory diseases accurately. They propose a hybrid architecture that is based on transfer learning. This architecture includes the integration of VGG16, a deep CNN as well as a 3D shallow CNN. The convolution layers used are depth-wise separable and the pooling module utilized is the Spatial pyramid pooling. An optimal blend of a pretrained 2D deep CNN and a 3D shallow CNN ensures effective usage of nuanced synergies among multivariate activation maps, resulting in more insightful patterns and, as a result, allowing more efficient learning techniques for the entire network. Their plan was to integrate into a shallow $3 \mathrm{D} \mathrm{CNN}$ all the $2 \mathrm{D}$ parameters learned from a 2D CNN, which would provide more contextual details and speed up the training process. Around 754 Covid-19 positive CXRs, 1341 negative CXRs, and 1345 Pneumonia positive CXRs were taken into account. 
Fig. 1 Depiction of the Block diagram of the system

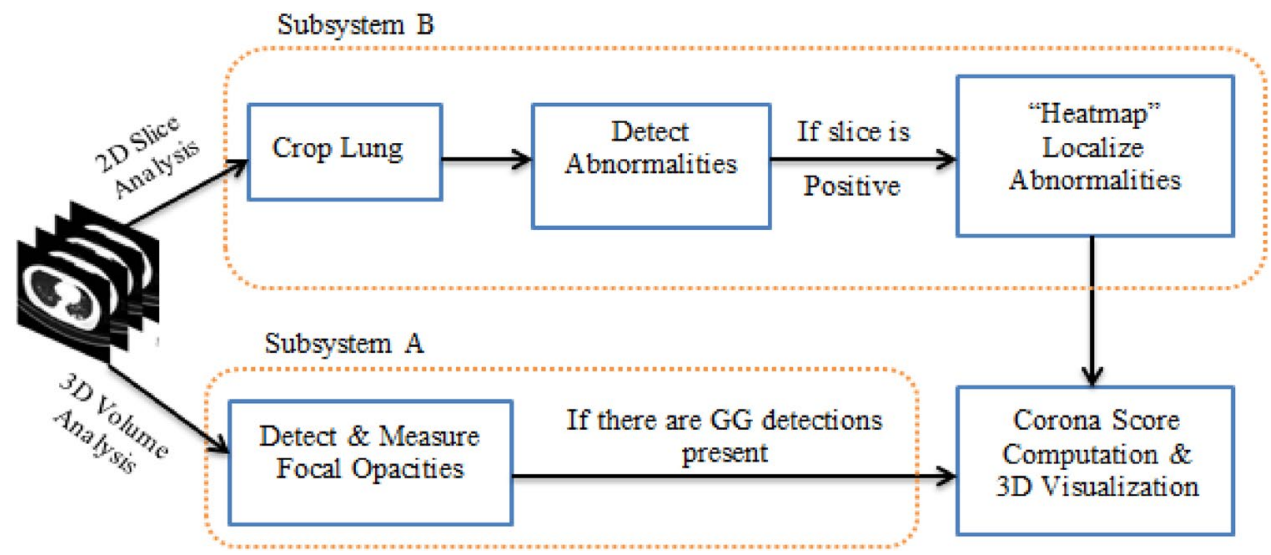

The Hybrid-Covid achieved a better balance of accuracy and computational complexity. The 3D CNN proposed is made up of three convolution layers that are implemented with different kernel sizes. This 3D CNN network can accommodate features at various scales by using one path for spatial information and another for wider contextual information. Three separate experiments were performed to assess and verify the proposed method's efficiency. In the baseline experiment, three collections yielded a total of 3440 CXR images. In this study, the Grad-CAM is used as a method to predict the class-discriminative localization map of an area affected in an apparently irrational manner. A second experiment was performed to further validate and test their architecture's predictive efficiency. The aim of this policy was to ensure that the models' discrimination abilities were not harmed as a result of the inclusion of sample artefacts and outliers in the data observations. The third experiment was carried out to see how the hybridization pipeline affected classification performance. The proposed method's results illustrate its effectiveness with an accuracy of $96.91 \%$, specificity of $98.68 \%$ and sensitivity of $98.33 \%$.

\section{Multi-Task Deep Learning Approaches for COVID-19 Detection}

With this implementation [5], numerous models are employed for performing a number of diverse tasks. An Inception Recurrent Residual Convolutional Neural
Network (IRRCNN) model with transfer learning is taken advantage for the detection task for this disease. A NABLA-N network is utilized for the purpose of segmentation of regions that are infected by the virus from CT images and X-ray. The researchers have employed the IRRCNN model and have also used X-ray images of the chest to classify normal cases from pneumonia cases. The IRRCNN model is employed to retrain their model to tell apart from Covid-19 and regular images in chest radiographs. They have also used 5 Inception Recurrent Residual Units (IRRUs). This system has also been tested with absolutely novel X-ray samples that were gathered from patients affected by Covid- 19 recently. This was done after successfully training the model for the detection of Coronavirus.

To spot Covid-19 affected regions in the chest radiographs, a NABLA-N model was used for the segmentation of regions of the chest alone. For carrying out refinement and choosing suitable contours for chest region extraction, mathematical morphological approaches were used. As for the feature extraction, an adaptive thresholding approach and a conventional image processing method were deployed. This was accomplished for detecting the regions infected by Coronavirus disease from the segmented chest section as shown in Fig. 2. The output of the heatmap image with respect to infected regions of Covid19 is also depicted in Fig. 2.
Fig. 2 The pipeline processing for the infected region detection-Chest Regions [5] and depiction of Coronavirus infected region detection: Mask for COVID-19 infected regions and final heatmap image
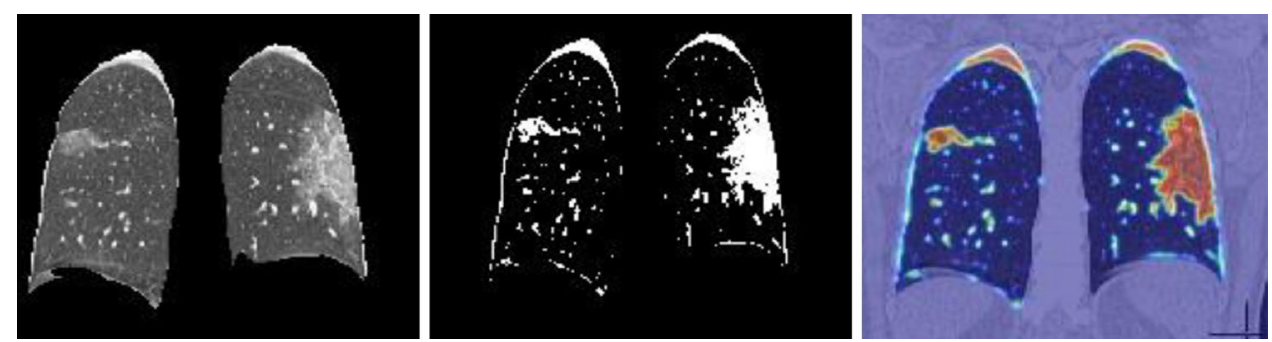

SN Computer Science a SPRINGer NATURE journal 
COVID-Det_CT: Covid-19 Detection and COVID-Seg_CT: Covid-19 Segmentation

The evaluation framework used by specialists in Wuhan, China, is used as a base to perform the task of detection from CT scans. The IRRCNN classification model is used for COVID-Det_CT, wherein it is actually trained using datasets gathered by the authors. A similar methodology is used for Covid-19 detection using X-ray images. They have also created and built an overarching system for the partitioning of lung regions, that is, for segmentation. This lung segmentation from 2D images is done for mining-specific infected regions in the lungs. The segmentation of chest $\mathrm{X}$-rays is shown in Fig. 3. Here, the unchanged NABLA-N model is employed which is as stated for Covid-19 Segmentation using X-ray images.

\section{Architecture Details}

The IRRCNN model deployed in this paper composes of an initial layer called the Input layer, 5 Inception Recurrent Residual Units: IRRUs, a GAP_Global Average Pooling layer and an output layer with the Softmax function. A $1 \times 1$ and $3 \times 3$ kernels for IRRU are used in this model [5]. This entire model employs approximately about 34 million network parameters. But, however, the NABLA-N network consists of three decoding units and an encoding unit.

This Pneumonia and Covid-19 detection method proposed in this paper presented up to $87.26 \%$ accuracy in testing. Their model based on IRRCNN also presented an improved accuracy of $3.76 \%$ in testing for the tasks of pneumonia detection. Many of the approaches recommended for the identification of the infected regions by COVID-19 are focused on patch-based methods of detection for extraction of infected regions. In this case, there is a high risk
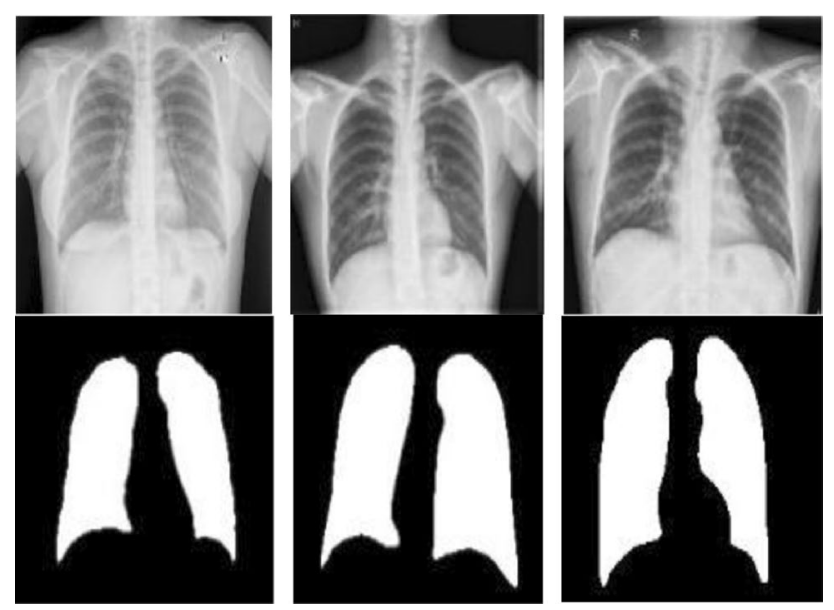

Fig. 3 Segmentation Chest X-ray [5] of detections being as false positives and false negatives as the pronouncements are made established on the class. The outcomes in their research evidently reveal the proficient detection of infected regions within the parts of the lungs. Therefore, the projected COVID-19 detection considerably diminishes the likelihood of false negative and false positive Covid-19 detections.

Other researchers have also contributed to the analysis of Covid-19 using Multi-task deep learning techniques. In [16], the authors have presented a classification segmentation tool to screen the Covid-19 using CT scans. They also propose a novel model that can both identify if a patient is positive and also segment the lesions of Covid-19 from the chest CTs. They employ three different tasks for learning called classification, segmentation and reconstruction simultaneously but use datasets that are different from each other. Their model's architecture comprised of an encoder, two decoders and a multilayer perceptron for the tasks of reconstruction, segmentation and classification. The major advantage of multitask learning is that it can combine several pieces of information from various tasks which in turn lead to better performance and generalization of the model. The first two tasks of classification and segmentation were mandatory while the last task of reconstruction was conducted to enhance the feature representation. To share parameters between the various tasks, they have used hard parameter sharing. The encoder and decoders that they have utilized is grounded on the architectures of U-Net. The encoders use a block of convolution and a skip connection follows it. Each decoder layer starts with an unsampling layer and then a convolution layer to have a two-fold reduction in the number of features. To evaluate their model, they performed three experiments in which the first experiment included tuning of hyper-parameters. The second experiment included a comparison of their model with the U-Net architecture to evaluate its performance on the segmentation task. The third experiment included a comparison of various models like VGG-16, ResNet50, VGG-19 Inception V3 etc., with their model on the classification task. They obtained a dice coefficient of $88 \%$ along with $99.7 \%$ specificity and $90.2 \%$ sensitivity for segmentation and accuracy of over $94 \%$ for classification.

\section{Customized Deep CNNs for Detection of Covid-19 Cases from Chest X-ray}

The study in [6] demonstrates a combined machine-human design approach utilizing a network called the COVID-Net. A publicly accessible benchmarked dataset was created and this dataset is referred to as "COVIDx". This facilitates the process of training and assessing their network, COVID-Net. The COVIDx employed to train and assess the suggested COVID-Net encompasses approximately 13,870 patient 


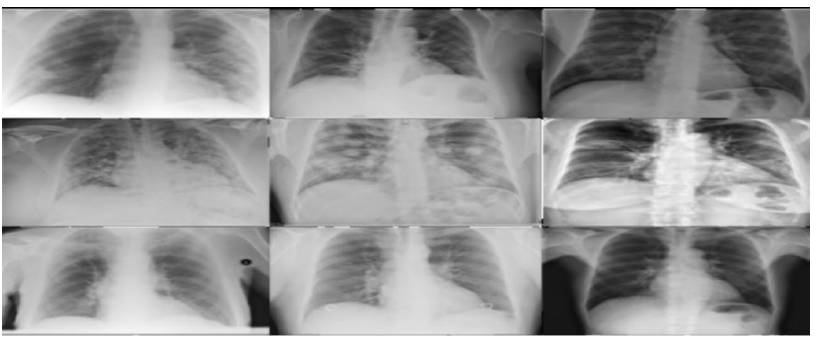

Fig. 4 Chest X-ray images belonging to COVIDx dataset. This dataset covers 13,975 Chest Radiographs through 13,870 cases gathered from 5 data repositories that are open source [6]

cases with a total of 13,975 images of CXR. Few example images belonging to this dataset is presented in Fig. 4.

In this research work, a prototype has been constructed preliminary to mark one among the three subsequent predictions: (i) normal- no infection, (ii) infections that are not COVID-19 and (iii) positive Covid-19 communicable infection. The logic for opting these probable predictions was because it could assist the doctors and clinicians to enhance the decision as to who should be ranked for Polymerase Chain Reaction testing for confirmation of cases but also in addition to what kind of treatment should be utilized depending upon the reason for infection.

The next stage of the machine-human collective plan for design which was engaged in developing the suggested COVID-Net was the design of the Machine-driven exploration stage. Precisely, in this particular stage, data behaves as a monitor to a design strategy besides the human-specific design requirements. This is supportive in identify and learning the ideal micro-architecture and macro-architecture designs. This can be still done while confirming that the domain-specific operational requirements are fulfilled by the Deep Neural Network architecture. In this paper, they combine the machine-driven design exploration strategy as a generative synthesis. This is centred on a sophisticated interaction amongst a pair of inquisitor-generator which works in line to gather intuitions and understandings. The suggested COVID-Net architecture of the network of this particular paper is depicted in Fig. 5.

\section{Lightweight Design Pattern}

Initial-stage Projection: $1 * 1$ filters or convolutions are used for proposing features of input to a much lower dimension. Depth-wise Representation: Effective depth-wise $3 * 3$ convolutions are used for understanding and learning spatial features to appropriately diminish the computational difficulty while conserving representational capacity. Expansion: $1 * 1$ filters or convolutions are used for escalating features to a greater dimension which is unlike than that of input features considered.

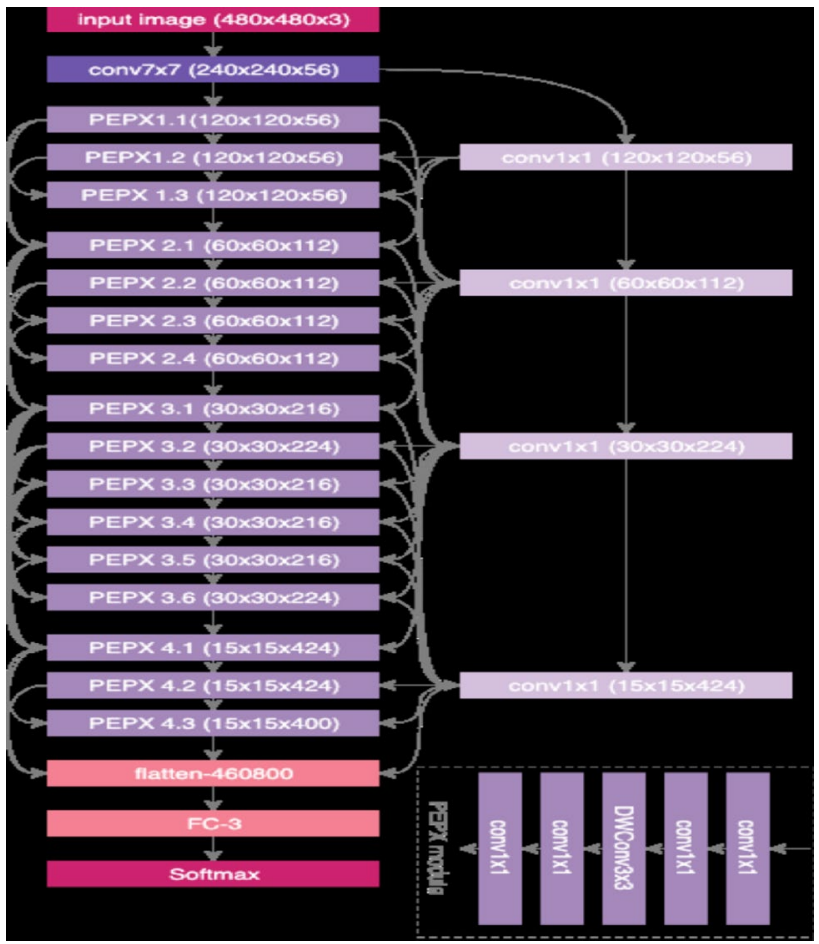

Fig. 5 Proposed COVID-Net Architecture of this paper [6]

Second-stage Projection: When projecting the features down to a lower dimension, convolutions or filters of $1 * 1$ are used. Extension: Convolutions or filters of $1 * 1$ are used that is finally used to extend and escalate the dimensionality of the channel to a dimension greater to yield eventual and ultimate features.

\section{Selective Long-Range Connectivity}

It can furthermore be seen that there is specific long-run connectivity in the architecture of COVID-Net at different architectural regions. The long-ranged connectivity utilization was observed while empowering improved representational limit. This is just to make the training simpler with commendable architectures of the network.

\section{Architectural Diversity}

A substantial assortment can also be seen in the architecture of COVID-Net. Specifically, this network architecture includes a diverse blend of convolution layers with a decent variety of sizes of kernels which ranged from $1 * 1$ to $7 * 7$.

Furthermore, they have also explored how COVID-Net could make predictions by means of explainable methods in efforts to obtain profound visions into critical factors that are associated with the cases of Covid-19.

Likewise in [17], the authors have presented dense convolutional networks based on image classifiers to categorize 
the X-ray images into three groups: Normal, Covid-19 and Pneumonia. The first network that they have used is referred to as the CheXNet which is a dense model having 121 layers. This model was priorly pre-trained on ImageNet and was also trained on the dataset of ChestX-ray 14 provided by NIH. Therefore, their CheXNet was trained twice and was retrained on a smaller dataset that was created which consisted of Covid-19, Pneumonia and normal X-rays. After successfully training the dense neural networks, they applied LRP-Layer wise relevance propagation followed by generation of heatmaps of X-rays considered.

They have also proposed a novel addition to their proposed technique of twice transfer learning called output neuron keeping. To keep these output neurons, a modest method available in PyTorch copies their weights and biases at the end of training the model on the second dataset. Finally, the DNNs output layer is changed to match the format of the desired output by finding out the neurons that would classify the classes similar to that when trained using the second dataset and then substitute their weights and biases respectively.

They trained five DNNs in which the first network, Network A was a 201 layered DenseNet, Network B was also a DenseNet201 that was trained using twice transfer learning. Network $\mathrm{C}$ was similar to Network B but with a modification of using the output neuron keeping method. Network D was a 121 layered DenseNet that used twice transfer learning as well as the output neuron keeping technique with only one output neuron. Network C, D and E achieved a perfect accuracy of $100 \%$, Network B achieved an accuracy of $98.7 \%$ while Network A obtained $99.3 \%$ accuracy.

\section{Al and ML in SARS-CoV-2 Prediction and Forecasting}

In this particular work [8], a model is created that could predict and forecast the infection of Covid-19 one to six days prior to the appearance of the symptoms. The authors have used a statistical analysis model called the Autoregressive Integrated Moving Average (ARIMA), Random Forest (RF), a rule-based Cubist Regression (CUBIST) model, StackingEnsemble Learning, Ridge Regression (RIDGE) and Support Vector Regression (SVR). When Stacking-Ensemble Learning approach is considered, the Random Forest, CUBIST regression, SV- and RIDGE models are considered as Base-learners and the Meta-Learner is the GP, Gaussian process. The accumulated dataset used for this analysis represents the jointly reported Covid-19 cases which existed in Brazil before mid of April 2020. An API was used to obtain the data for the same. States with low temperatures, south Brazilian states and ten states having a high occurrence of positive cases were selected and considered.

A model based on Box \& Jenkins modelling is called ARIMA. When time series that was non-stationary was to be considered, this model proved the best to be used. This model is completely specified by trend differences of different degrees $(d)$, autoregressiveness $(p)$ and moving average operators $(q)$. The ARIMA model used in this paper is designated as $\operatorname{ARIMA}(p, d, q)$ [9]. GP, Gaussian Process encompasses random variable sets that are Gaussian distributed. They are wholly identified by its mean and kernel or covariance function [10]. The model CUBIST performs forecasting and predictions using the principle regression of trees. This is a rule-based model [11]. The regularized regression approach referred to as RIDGE, engages a term for penalization in the conventional LS algorithm, the leastsquares algorithm [12]. RF, Random Forest is a model that associates the bagging benefits which is characterized by the formation of numerous samples. This is done through refitting of the bootstrap technique; that is arbitrary choice of predictors to make up individual nodes of decision tree from the same set of data [13]. SVR, Support Vector Regression involves determining the support vectors or points that are close and neighbouring to a hyperplane which could maximize the margins amongst two-point classes that were attained from the difference between the threshold and target value. Stacked Generalization, an ensemble dependent approach [14] syndicates the predictions of base learners which are a set of weak models through a meta-learner, to acquire a strong learner. The initial stage begins by splitting the input data into two sets, specifically, the training and test datasets. The last six observations are put under testing dataset and the remaining samples are considered to be the training set. The training data are distributed by its standard deviation and focused by its mean value. The base-learners such as the RF, CUBIST, SVR and RIDGE are trained when the Stacking Ensemble Learning model is employed. The predictions are then considered as inputs for the GP, which is a meta-learner. The Leave-One-Out Cross validation along with a time slice is implemented during the training stage. Figure 6 represents the proposed framework used for forecasting and predictions.

With the results obtained by the authors, it was inferred that Stacking-Ensemble learning model and SVR were the most apt tools for predicting and forecasting the cases of COVID-19 for most of the Brazilian states considered for the analysis. These models and approaches were also able to pick up the nonlinearities that were innate to the assessed epidemiological series of time. Furthermore, the ARIMA model can also be well-thought-out in some aspects when predictions are done one day ahead. The RIDGE and CUBIST models are worthy of consideration while developing prediction tasks in time windows of 'three days ahead' and 'seven days ahead' predictions. The authors for each model obtained a best symmetric mean absolute percentage error that ranged between $0.87 \%$ to $3.51 \%$ for 'one day ahead' predictions, $1.02 \%$ to $5.63 \%$ for 'three days ahead' predictions 
Fig. 6 Depicts the forecasting and prediction framework proposed [8]
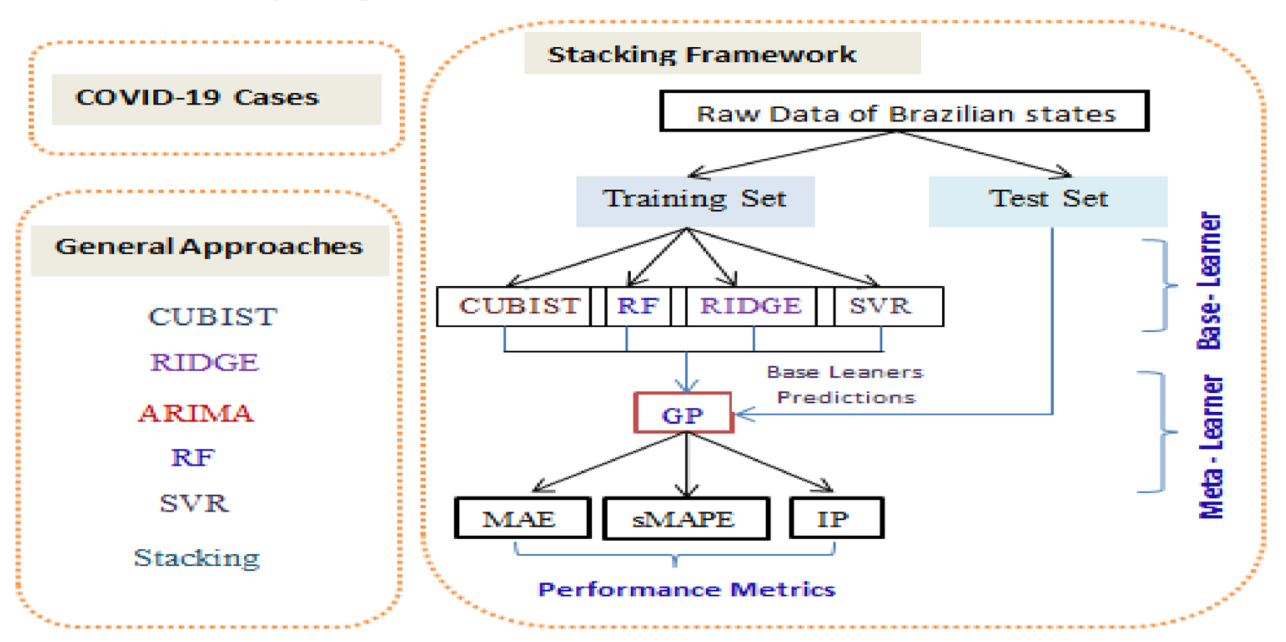

and $0.95 \%$ to $6.90 \%$ for 'seven day ahead' predictions. Thus, the classification of models from finest to substandard when precision and accuracy were considered is: Support Vector Regression, Stacking-Ensemble Learning, ARIMA, CUBIST, RIDGE, and RF models.

Similarly in [18], the authors proposed a model based on Machine Learning that predicted Covid-19 infection in the data obtained by using eight basic questions. The Israeli Health ministry made data of people who tested positive for Covid-19 public. The dataset contains daily preliminary records of all residents who were screened for Covid-19 throughout the country. There are also clinical indications, sex, and a binary measure of whether the person being tested is 60 years old or older, in addition to the test date and results. Using the above data, they designed a model that used features like age, gender, and clinical symptoms like fever, cough, and headache to forecast test results. The training and test set used included records of 51,831 individuals who were tested of which there were 4769 confirmed positive cases. The predictions were made using a gradient-boosting model having decision tree base learners. The gradient-boosting predictor dealt with missing values inherently. SHAP values, SHapley Additive exPlanations were determined to classify the core features that drive model prediction. Complicated models, such as ANNs; Artificial
Neural Networks and Gradient-Boosting machines benefit from these principles. SHAP values that originated from game theory split each sample's prediction outcome into the contribution of each constituent function value. This can be achieved by matching models with subsets of function space and calculating variations between them. SHAP values approximate the impact of individual function to overall the model predictions by averaging through samples.

This model predicted with 0.90 area under the Receiver Operating Characteristic curve, auROC with an accuracy of 95\% for the prospective test range. The potential working points based on estimates from the test range are $87.30 \%$ sensitivity and $71.98 \%$ specificity, or $85.76 \%$ sensitivity and $79.18 \%$ specificity. Not only do the authors recognize that self-reporting of symptoms is often skewed, they also stress the necessity for more reliable data that could supplement their system. As a better understanding of the role of different symptoms in diagnosing the disease develops, more indications may be added to future models.

\section{Datasets Available Online}

See Table 1.

Table 1 Available open- source datasets

\begin{tabular}{ll}
\hline Sources & Data Type \\
\hline 1. UCSD-AI4H & CT images [https://github.com/UCSD-AI4H/COVID-CT] \\
2. Kaggle & X-ray and CT images [https://www.kaggle.com/bachrr/covid-chest-xray] \\
3. The Johns Hopkins University & Web-based mapping global cases [https://systems.jhu.edu/research/public-health/ncov/] \\
4. (BSTI) The British Society of Thoracic Imaging & Chest X-ray and CT images [https://www.bsti.org.uk/training-and-education/covid-19- \\
& bsti-imaging-database/] \\
5. Italy Ministry of Health & Cases in Italy [https://github.com/pcm-dpc/COVID-19] \\
\hline
\end{tabular}




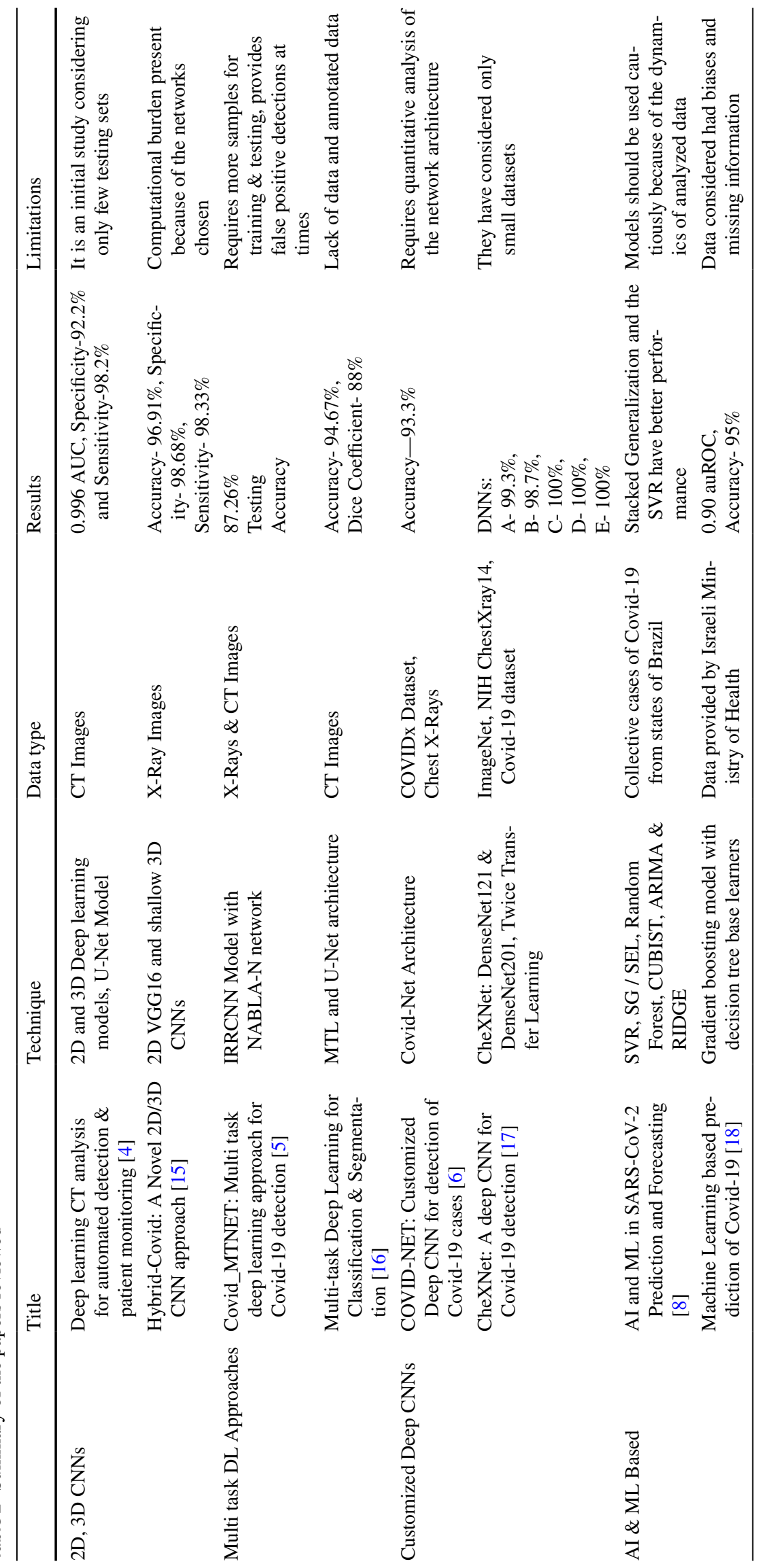




\section{Discussion}

The major focus of this particular work was to review the prime Deep Learning and Machine Learning techniques to detect Covid-19 using various datasets. The summary of the works considered in this paper is presented in Table. 2. It can be observed that most of the works are carried out using $\mathrm{CT}$ images and $\mathrm{X}$-rays as they are the most widely available data.

\section{Conclusion and Future Scope}

The deadly COVID-19 epidemic has greatly impacted people's lives across the world. In this study, we comprehensively review various Covid-19 detection techniques using ML and DL that can help clinicians and doctors in recognizing the Covid-19 cases in a short span of time. The various Deep Learning techniques that we reviewed included 3D and 2D analysis of the chest CT images a Hybrid-Covid network, an IRRCNN model with NABLA-N network and CovidNet and CheXNet network architecture based detection. The accuracy of the CheXNet architecture-based detection had the highest accuracy. The Machine Learning approaches employed various models like RF, ARIMA, SVR, CUBIST, and Gradient Boosting to precisely make predictions. It was found that the SVR and SEL were the best in accuracy terms. Using data of chest radiology visualization is prevalent as it seems prevalent among the reported works. The future scope in this region of Covid-19 detection is to use various other algorithms and techniques like IRCNN, Multi-Criteria Decision Analysis and also Artificial Intelligence-powered Internet of Things applications that can well in advance detect Covid-19 with ease.

\section{Declarations}

\section{Conflict of interest}

The authors declare that they have no conflict of interest.
2. Chan JF, et al. A familial cluster of pneumonia associated with the 2019 novel coronavirus indicating person-to-person transmission: a study of a family cluster. Lancet. 2020;395(10223):514-23.

3. Zu ZY et al. Coronavirus disease 2019 (COVID-19): a perspective from China. In: Radiology, p. 2004902020.

4. Ophir G et al. Rapid AI development cycle for the coronavirus (COVID-19) pandemic: initial results for automated detection \& patient monitoring using deep learning $\mathrm{CT}$ image analysis. In: Radiology- Artificial Intelligence, arXiv:2003.05037 [eess.IV] 2020.

5. Md Zahangir A et al. COVID_MTNet: COVID-19 detection with multi-task deep learning approaches. In: arXiv:2004.03747 [eess. IV], 2020.

6. Linda $\mathrm{W}$ et al. Covid-Net: a tailored deep convolutional neural network design for detection of covid-19 cases from chest X-ray. In: arXiv:2003.09871v4 [eess.IV], 2020.

7. Mucahid, B et al. Coronavirus (Covid- 19) classification using CT images by machine learning methods. In: arXiv:2003.09424, 2020.

8. Matheus H, Dal Molin Ribeiro et al. Short term forecasting Covid19 cumulative confirmed cases: perspectives for Brazil. In: Chaos Solit Fract. https://doi.org/10.1016/j.chaos.2020.1098532020.

9. Box, GE et al. Time series analysis- forecasting and control. In: 5th edition, Wiley \& Sons (2015).

10. Rasmussen, CE. Gaussian processes in machine learning. In: Springer, pp. 63-71, Heidelberg, 2004.

11. Quinlan JR. Combining instance-based and model-based learning. In: Proceedings of the 10th international conference on international conference on machine learning, Morgan Kaufmann Publishers Inc. ICML'93, pp. 236-243, San Francisco, CA, 1993.

12. Hoerl AE, Kennard RW. Ridge regression- Biased estimation for non-orthogonal problems. Technometrics. 1970;12(1):55-67. https://doi.org/10.1080/00401706.1970.10488634.

13. Breiman L. Random forests in Machine. Learning. 2001;45(1):532. https://doi.org/10.1023/A:1010933404324.

14. Wolpert DH. Stacked generalization. Neural Network. 1992;5(2):241-59. https://doi.org/10.1016/S0893-6080(05) 80023-1.

15. Bayoudh K, et al. Hybrid-COVID: a novel hybrid 2D/3D CNN based on cross-domain adaptation approach for COVID19 screening from chest X-ray images. Phys Eng Sci Med. 2020;43:1415-31.

16. Amine A et al. Multi-task deep learning based CT imaging analysis for COVID-19 pneumonia: Classification and segmentation. In Computers in Biology and Medicine, Volume 126, 2020.

17. Pedro R, Bassi AS, Romis A: A deep convolutional neural network for COVID-19 detection using chest X-rays. In: arXiv:2005.01578v4 [eess.IV], 2021.

18. Zoabi Y, Deri Rozov S, Shomron N. Machine learning-based prediction of COVID-19 diagnosis based on symptoms. In npj digital medicine, volume 4, 2020.

Publisher's Note Springer Nature remains neutral with regard to jurisdictional claims in published maps and institutional affiliations.

\section{References}

1. Wang et al. Clinical characteristics of 138 hospitalized patients with 2019 novel coronavirus- infected pneumonia in Wuhan, China. In: Jama, p. in Press, 2020. 